\title{
Interstellar atoms, molecules and diffuse bands toward SN2006X in M 100^
}

\author{
N. L. J. Cox ${ }^{1}$ and F. Patat ${ }^{2}$ \\ ${ }^{1}$ Herschel Science Centre, European Space Astronomy Centre, ESA, PO Box 78, 28691 Villanueva de la Cañada, Madrid, Spain \\ e-mail: nick.cox@sciops.esa.int \\ ${ }^{2}$ European Southern Observatory, K. Schwarzschild Str. 2, 85748, Garching b. München, Germany
}

Received 14 March 2008 / Accepted 6 May 2008

\section{ABSTRACT}

\begin{abstract}
Aims. Supernovae offer the unique possibility to probe diffuse extra-galactic sightlines via observation of the optical transitions of atoms, molecules and the diffuse interstellar bands (DIBs). Through optical spectroscopy the presence of (complex) molecules in distant galaxies can be established and used to derive local physical conditions of the interstellar medium (ISM).

Methods. High resolution optical (3300-6800 Å) spectra of SN2006X at different phase obtained with UVES on the VLT were reduced and analysed.

Results. In addition to previously detected atomic (Na I and $\mathrm{Ca}$ II) and molecular (CN) transitions we present detections of DIBs $(\lambda \lambda 6196,6283)$, diatomic molecules $\left(\mathrm{CH}, \mathrm{CH}^{+}\right)$and neutral atoms $(\mathrm{Ca} \mathrm{I})$ in the spectra of SN2006X taken at different phases (at 2 days before and 14 and 61 days after the brightness maximum). An analysis of the absorption profiles shows no variation between phases in the abundance, nor the central velocities (within $3 \sigma$ error bars) of the (dense) gas tracers $\left(\mathrm{CH}, \mathrm{CH}^{+}\right.$and $\mathrm{Ca}$ I) and the DIBs. This is consistent with the conclusion in the literature that SN2006X exploded behind a dense interstellar cloud (inferred from strong atomic sodium and calcium lines and $\mathrm{CN}$ transitions) which caused strong photometric reddening but whose material was not directly affected by the supernova explosion. The $\mathrm{CH}$ and $\mathrm{CN}$ column densities correspond to a reddening of one magnitude following the Galactic correlation derived previously. The $\lambda \lambda 6196$ and 6283 lines detected in the M 100 ISM are under-abundant by factor of 2.5 to 3.5 (assuming a visual extinction of $\sim 2 \mathrm{mag}$ ) compared to the average Galactic ISM relationship. Upper limits for $\lambda \lambda 6379$ and 6613 show that these are at least a factor of seven weaker. Therefore, the Galactic DIB-reddening relation does not seem to hold in M 100, although the lower gas-to-dust ratio may further reduce this discrepancy.
\end{abstract}

Key words. ISM: lines and bands - ISM: molecules - ISM: dust, extinction - ISM: clouds - ISM: individual objects: M 100

\section{Introduction}

Optically bright supernovae ( $\mathrm{SNe}$ ) offer the unique possibility to probe the diffuse interstellar medium (ISM) in galaxies beyond those of the Local Group. Despite the serendipitous nature of supernovae events, the study of their high-resolution optical spectra for interstellar lines has seen a number of successes in recent years. Optical studies of narrow interstellar absorption lines and diffuse interstellar bands superimposed on the continuum and broad line spectra of supernovae started in earnest in the late 1980s. First, Rich (1987) detected strong sodium lines and tentatively four diffuse interstellar bands (DIBs) toward SN1986G in NGC 5128. D'Odorico et al. (1989) confirmed the presence of DIBs toward SN1986G and reported on the detection of $\mathrm{CH}$ and $\mathrm{CH}^{+}$. SN1987A provided a unique opportunity to observe interstellar lines and diffuse interstellar bands in the LMC (Vidal-Madjar et al. 1987; Vladilo et al. 1987; Welty et al. 1999). Steidel et al. (1990) detected sodium, calcium and diffuse interstellar bands toward SN1989M in NGC 4579. Complex sodium and calcium profiles were observed toward SN1993J in M 81 but no DIBs or molecules were detected (Vladilo et al. 1994; Benetti et al. 1994). Sollerman et al. (2005) detected and resolved most of the prominent diffuse interstellar bands toward SN2001el in the spiral galaxy NGC 1448. Their high-resolution

* Based on observations with VLT-UVES under Run IDs 276.D-5048, 277.D-5003 and 277.D-5013 and CFHT-ESPaDOnS run 05ao5. spectra showed a remarkable similarity (both in relative strength and profile) between DIBs observed in this distant ( 15 Mpc) galaxy and the Milky Way. GRBs and DLAs have been used to study the ISM in even more distant systems (e.g. Ellison et al. 2007; Lawton et al. 2007). In contrast, the most distant galaxy probed by direct observations of early-type supergiants is M 31 (Cordiner et al. 2008).

The spectra discussed in this paper were previously investigated in the context of the presence and evolution of circumstellar material of supernovae (Patat et al. 2007). Motivated by the report of Lauroesch et al. (2006) on strong interstellar features we further exploit these spectra to characterise and analyse the ISM of M 100 probed by SN2006X. First, we give a brief overview of relevant data on SN2006X (Sect. 2) and outline the observations (Sect. 3). Next, we present the detected (di)atomic lines and DIBs and derive column densities and equivalent widths (Sect. 4). The results and implications are discussed in Sect. 5.

\section{SN2006X}

SN2006X is a normal type Ia supernova that exploded within or behind the disk of the host galaxy M 100 (also known as NGC 4321) in the Virgo cluster. Although normal, it does show some distinct properties (e.g. very high expansion velocities, peculiar colour evolution) that may be characteristic of type Ia 
Table 1. Observational log for SN2006X with UVES. The phase is given in days with respect to the $B$-band maximum light (February 20, 2006). The UVES setting simultaneously covered three wavelength

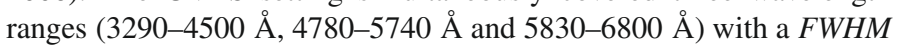
resolution of $\sim 7 \mathrm{~km} \mathrm{~s}^{-1}$.

\begin{tabular}{llll}
\hline \hline $\begin{array}{l}\text { UT Date } \\
(\mathrm{dd} / \mathrm{mm} / 2006)\end{array}$ & $\begin{array}{l}\text { Phase } \\
(\text { days })\end{array}$ & $\begin{array}{l}\text { Exp. Time } \\
(\text { seconds })\end{array}$ & $\begin{array}{l}\text { Heliocentric correction } \\
\left(\mathrm{km} \mathrm{s}^{-1}\right)\end{array}$ \\
\hline $18 / 02$ & -2 & 4175 & +14.6 \\
$06 / 03$ & +14 & 8940 & +7.2 \\
$22 / 04$ & +61 & 15025 & -15.4 \\
\hline
\end{tabular}

supernovae arising in young and dusty environments (Wang et al. 2008). The host galaxy recession velocity is $1571 \mathrm{~km} \mathrm{~s}^{-1}$ (at a distance of $15.2 \mathrm{Mpc}$ ) whereas the component of the rotation velocity along the line of sight at the apparent SN location is about $75 \mathrm{~km} \mathrm{~s}^{-1}$ (Rand 1995; Kuno et al. 2007). The latter coincides approximately with the strongly saturated $\mathrm{Na}$ I D and Ca II H\&K components and a weakly saturated $\mathrm{CN}$ vibrational band. These strong components, unaffected by the supernova event, originate from an interstellar molecular cloud (or system of clouds) within the disk of M 100 at some distance in front of SN2006X (Patat et al. 2007). Wang et al. (2008) derive, via four different methods, a strong reddening of $E_{B-V}=1.42 \pm 0.04$ mag (assuming a Galactic foreground extinction of $0.026 \mathrm{mag}$ ).

\section{Observations and spectra}

SN2006X was observed with the Ultraviolet and Visual Echelle Spectrograph (UVES; Dekker et al. 2000) mounted at the European Southern Observatory (ESO) $8.2 \mathrm{~m}$ Very Large Telescope (VLT). Observations were carried out at four different phases with respect to maximum light (20 February 2006) in the $B$-band (Table 1; see Patat et al. 2007).

For UVES the 390-580 setup was used, which is unfortunate since it is notorious for not covering the best studied DIBs at 5780 and $5797 \AA$ (even in this case where they are redshifted by about $32 \AA$ ). UVES data were reduced with the UVES data reduction pipeline software (Ballester et al. 2000). HIRES and UVES spectra of SN2006X were taken at phase +105 and +121 days, respectively. However, these spectra have insufficient signal-to-noise for the purpose of our analysis of weak narrow features and are therefore not further considered in this letter. The spectral $F W H M$ velocity resolution is $6.6-7.3 \pm 0.7 \mathrm{~km} \mathrm{~s}^{-1}$ (from blue to red) and the uncertainty in the ThAr wavelength calibration is $0.15 \mathrm{~km} \mathrm{~s}^{-1}$. To correct for the Earth's motion a heliocentric velocity correction (Table 1) has been applied to the individual exposures.

\section{Interstellar atoms, molecules and diffuse bands}

The $\mathrm{CaI}, \mathrm{CH}^{+}$and $\mathrm{CH}$ transitions were fitted with the VPFIT $\operatorname{code}^{1}$. The resulting central velocities and column densities are given in Table 2 for $\mathrm{Ca} \mathrm{I}, \mathrm{CH}^{+}$and $\mathrm{CH}$. VPFIT uncertainties depend directly on the line parameters (width and strength) and the $\mathrm{S} / \mathrm{N}$. The smallest Doppler widths $\left(\sim 1 \mathrm{~km} \mathrm{~s}^{-1}\right)$ indicate that these lines are not, or only marginally, resolved. The small shift in velocity between $\mathrm{Ca}$ I and $\mathrm{CH}^{+} / 6196$ DIB is notable but probably systematic due to their relative weakness and presence of

\footnotetext{
1 VPFIT (version 9.3) has been developed by R. F. Carswell, K. Webb, M. J. Irwin, and A. J. Cooke. It is available at http://www. ast. cam. ac.uk/ rfc/vpfit.html
}

Table 2. Heliocentric corrected radial velocities $v\left(\mathrm{~km} \mathrm{~s}^{-1}\right)$, Doppler widths $b\left(\mathrm{~km} \mathrm{~s}^{-1}\right)$ and column densities $N\left(\mathrm{~cm}^{-2}\right)$ have been derived with VPFIT for CaI, $\mathrm{CH}^{+}$and $\mathrm{CH}$ transitions toward SN2006X. $1 \sigma$ rms-errors are given in parenthesis. $\mathrm{CH}$ at phase +61 is not well constrained (low $\mathrm{S} / \mathrm{N})$ but consistent with earlier phases. Contamination in $\mathrm{CH}^{+}(4232 \AA)$ at phase -2 and strong noise in $\mathrm{CH}^{+}(3957 \AA)$ at phase +14 made it impossible to properly constrain fits to this lines. Straightforward peak velocity and strength measurements do however agree with the other two transitions. For the $\mathrm{CN} \mathrm{R}(0)$ transitions $v$ is 1645 and $1647 \mathrm{~km} \mathrm{~s}^{-1}$ at phase -2 and +14 , respectively.

\begin{tabular}{llccc}
\hline \hline Transition & $\begin{array}{l}\text { Phase } \\
\text { (days) }\end{array}$ & $\begin{array}{c}v \\
\left(\mathrm{~km} \mathrm{~s}^{-1}\right)\end{array}$ & $\begin{array}{c}b \\
\left(\mathrm{~km} \mathrm{~s}^{-1}\right)\end{array}$ & $\begin{array}{c}\log N \\
\left(\mathrm{~cm}^{-2}\right)\end{array}$ \\
\hline $\mathrm{Ca}$ I & -2 & $1641(2)$ & $2(2)$ & $10.8(0.2)$ \\
& +14 & $1643(1)$ & $1(1)$ & $10.8(0.2)$ \\
$\mathrm{CH}^{+}$ & -2 & $1647(1)$ & $4(2)$ & $13.7(0.1)$ \\
& +14 & $1651(1)$ & $3(2)$ & $13.6(0.1)$ \\
$\mathrm{CH}$ & -2 & $1645(1)$ & $4(1)$ & $13.78(0.03)$ \\
& +14 & $1647(1)$ & $2(1)$ & $13.8(0.3)$ \\
& +61 & $1648(2)$ & $2(2)$ & $13.8(0.2)$ \\
\hline
\end{tabular}

Table 3. Radial heliocentric velocities and equivalent widths for $\lambda \lambda 6196$ and 6283 ( $1 \sigma$ rms-error in parenthesis). $3 \sigma$ (=3.0 FWHM $\left.\sigma_{\text {noise }}\right)$ upper limits for $\lambda \lambda 6379$ and 6613. Uncertainties in $\lambda 6283$ central velocity are of the order of 10 to $20 \mathrm{~km} \mathrm{~s}^{-1}$.

\begin{tabular}{lcccccc}
\hline \hline \multirow{2}{*}{$\begin{array}{l}\text { Phase } \\
\text { (days) }\end{array}$} & \begin{tabular}{c}
$c$ \\
\cline { 2 - 7 } \\
\cline { 2 - 7 } $\left.\mathrm{km} \mathrm{s}^{-1}\right)$
\end{tabular} & $\begin{array}{c}w \\
(\mathrm{~m} \AA)\end{array}$ & $\begin{array}{c}v \\
\left(\mathrm{~km} \mathrm{~s}^{-1}\right)\end{array}$ & $\begin{array}{c}w \\
(\mathrm{~m} \AA)\end{array}$ & $\begin{array}{c}3 \sigma \\
(\mathrm{m} \AA)\end{array}$ & $\begin{array}{c}3 \sigma \\
(\mathrm{m} \AA)\end{array}$ \\
\hline-2 & $1647(1)$ & $12(2)$ & $\sim 1677$ & $160(15)$ & $<13$ & $<31$ \\
+14 & $1650(1)$ & $13(2)$ & $\sim 1656$ & $180(15)$ & $<11$ & $<29$ \\
+61 & $1649(1)$ & $16(3)$ & $\sim 1639$ & $190(20)$ & $<25$ & $<55$ \\
\hline
\end{tabular}

noise artifacts near the interstellar features. The $\sim 2 \mathrm{~km} \mathrm{~s}^{-1}$ velocity difference between the first two epochs is probably due to a rigid shift of the UVES instrument between nights.

Template 6196, 6283, 6379 and $6613 \AA$ DIBs were constructed (via a multiple Gaussian profile fit) from high quality $(R \sim 80000, S / N \sim 600)$ spectra of the Galactic target $\beta^{1}$ Sco (HD 144217; $E_{B-V}=0.22 \mathrm{mag}$ ). The 6196 and 6283 template profiles were fitted to the observed spectra with intensity and central velocity set as free parameters. The 6379 and $6613 \AA$ DIBs are not detected, but upper limits on the strength (at the expected velocity) were derived. Central velocities and equivalent widths or $3 \sigma$ limits are reported in Table 3 . The central velocities of the $6283 \AA$ DIB are uncertain due to the width of the profile and the large uncertainty on its rest wavelength, $\lambda_{0}$.

\section{Discussion and conclusion}

The high quality (in particular high $\mathrm{S} / \mathrm{N}$ ) of the UVES data has made it possible to detect and analyse molecular lines and diffuse interstellar bands toward SN2006X probing the ISM of M 100.

The (dense) gas tracers $\mathrm{Ca}$, $\mathrm{CH}$ and $\mathrm{CH}^{+}$are detected in the spectra of SN2006X taken at phase -2 and +14 . $\mathrm{CH}$ has also been detected in the phase +61 spectrum. Non-detections for later phases are due to reduced $\mathrm{S} / \mathrm{N}$. Radial velocities and column densities remain invariant (within error bars) between the different phases. The derived $\mathrm{CH}$ column density is in good agreement, using the Galactic correlation (Weselak et al. 2004), with that derived from $\mathrm{CN}$ and Na I measurements, implying $E_{B-V} \sim 1$. The lower $\mathrm{CH}^{+}$abundance suggests a quiescent medium without the frequent shocks that are normally associated with $\mathrm{CH}^{+}$production (Crawford 1989; Falgarone et al. 1995). 


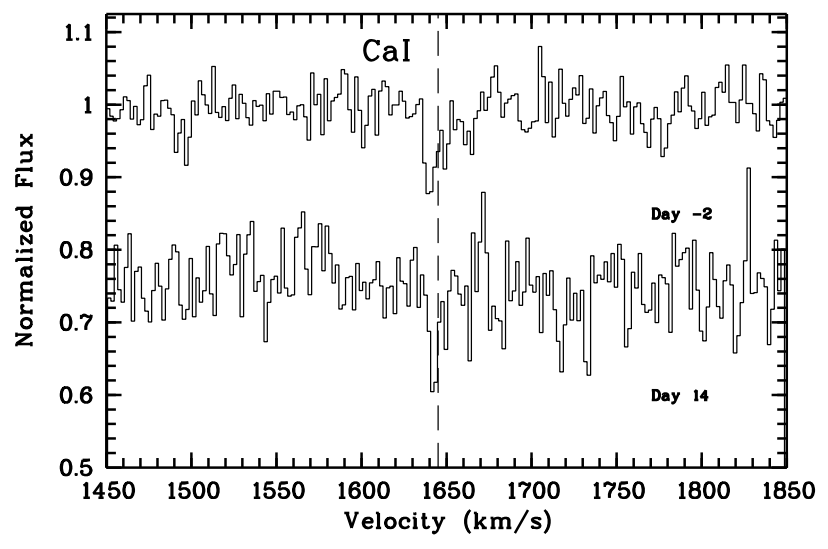

Fig. 1. The Ca I $\left(\lambda_{0}=4226.73 \AA\right)$ transitions toward SN2006X. Spectra are rebinned to $2 \mathrm{~km} \mathrm{~s}^{-1}$. Vertical dashed line at $v_{\text {helio }}=1645 \mathrm{~km} \mathrm{~s}^{-1}$.

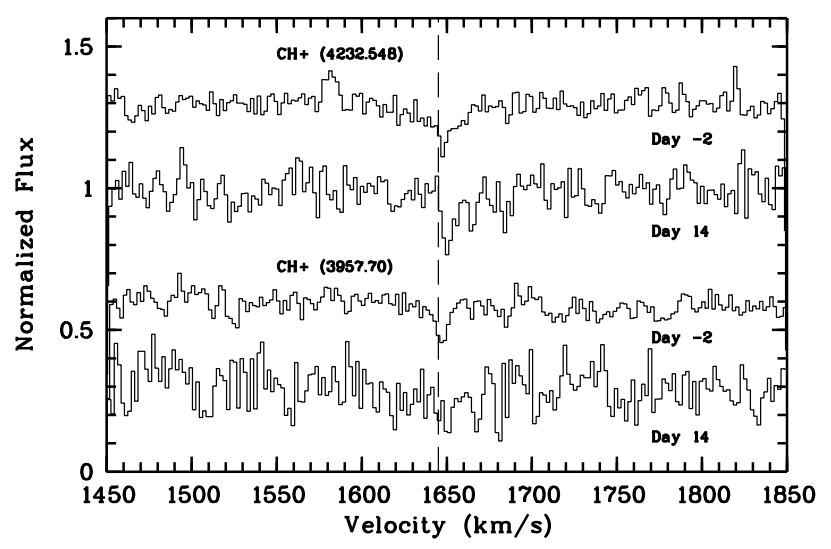

Fig. 2. The $\mathrm{CH}^{+}$(top two spectra with $\lambda_{0}=4232.548 \AA$ and bottom two spectra with $\lambda_{0}=3957.70 \AA$ ) transitions toward SN2006X. Spectra are rebinned to $2 \mathrm{~km} \mathrm{~s}^{-1}$. Vertical dashed line at $v_{\text {helio }}=1645 \mathrm{~km} \mathrm{~s}^{-1}$.

The equivalent widths of the detected DIBs are also invariant within the uncertainties. The observed $\lambda 6283$ is seven times weaker (adopting $E_{B-V}=1.4$ for SN2006X) compared to the average Galactic (MW) value, while the observed $\lambda 6196$ is about five times weaker. See Table 4 for a comparison of different Galactic and extra-Galactic lines-of-sight. The non-detected $\lambda \lambda 6379$ and 6613 are at least a factor of fifteen under-abundant. Since $\lambda 6196$ and $\lambda 6613$ are closely correlated in Galactic environments it is striking that $\lambda 6196$ is detected while the usually stronger $\lambda 6613$ is not. From the observed DIB strengths it is not possible to determine how much more under-abundant the 6379 and 6613 DIBs are with respect to the detected 6196 and 6283 DIBs (whose strength ratio is in the observed range). The overall weakness of DIBs toward SN2006 suggests that the general relation between DIB strength and reddening does not hold.

The inferred total-to-selective visual extinction ratio, $R_{V}$, of the dust toward SN2006X is 1.48 (Wang et al. 2008), much lower than the typical values observed for Galactic extinction: $R_{V}=$ $3.1 \pm 1.5$ (Fitzpatrick \& Massa 2007). Therefore, if we compare DIB strengths per unit visual extinction (with $A_{V}=R_{V} \cdot E_{B-V}$ ) the apparent under-abundances is reduced by a factor of two.

At this point a word of caution is warranted regarding the derived reddening from photometric observations. The presence of circumstellar light echos can affect the derived colours and

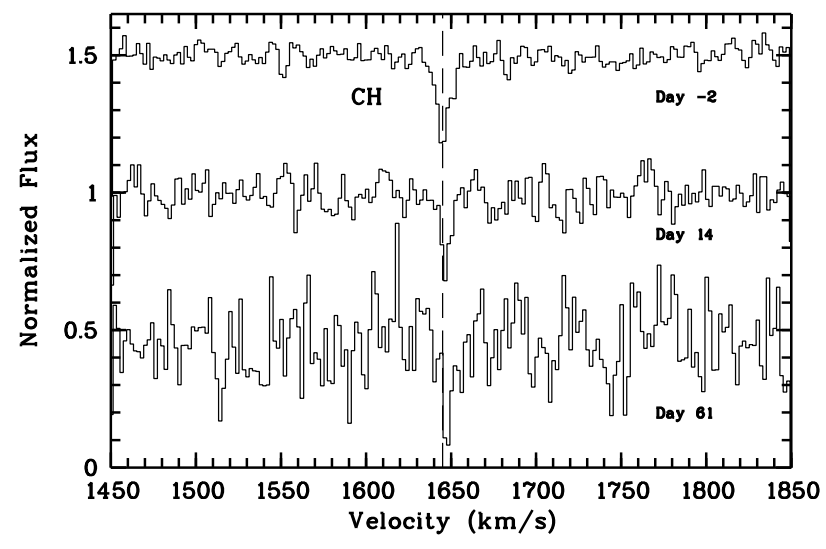

Fig. 3. The CH transition toward SN2006X $\left(\lambda_{0}=4300.303 \AA\right)$. Spectra are rebinned to $2 \mathrm{~km} \mathrm{~s}^{-1}$. Vertical dashed line at $v_{\text {helio }}=1645 \mathrm{~km} \mathrm{~s}^{-1}$.

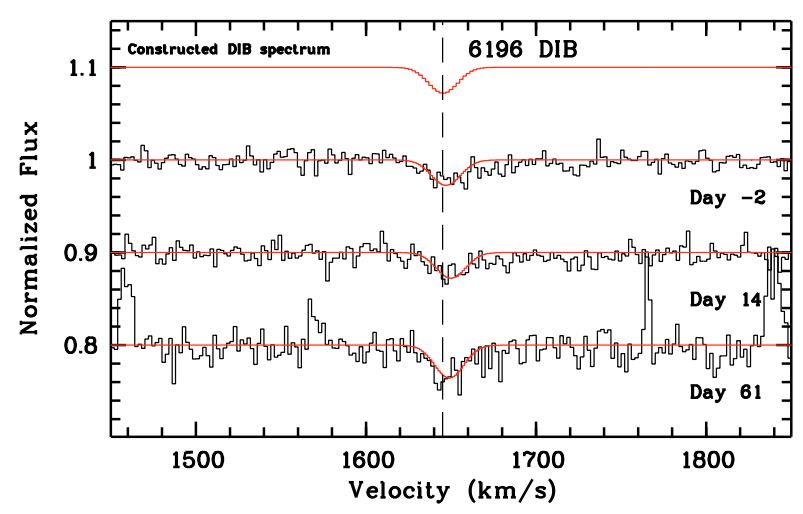

Fig. 4. The $6195.96 \AA$ DIB detection toward SN2006X. The reference spectrum (top) has been constructed from a Galactic 6196 band profile shifted to the SN2006X velocity. Spectra are rebinned to $2 \mathrm{~km} \mathrm{~s}^{-1}$. Vertical dashed line at $v_{\text {helio }}=1645 \mathrm{~km} \mathrm{~s}^{-1}$.

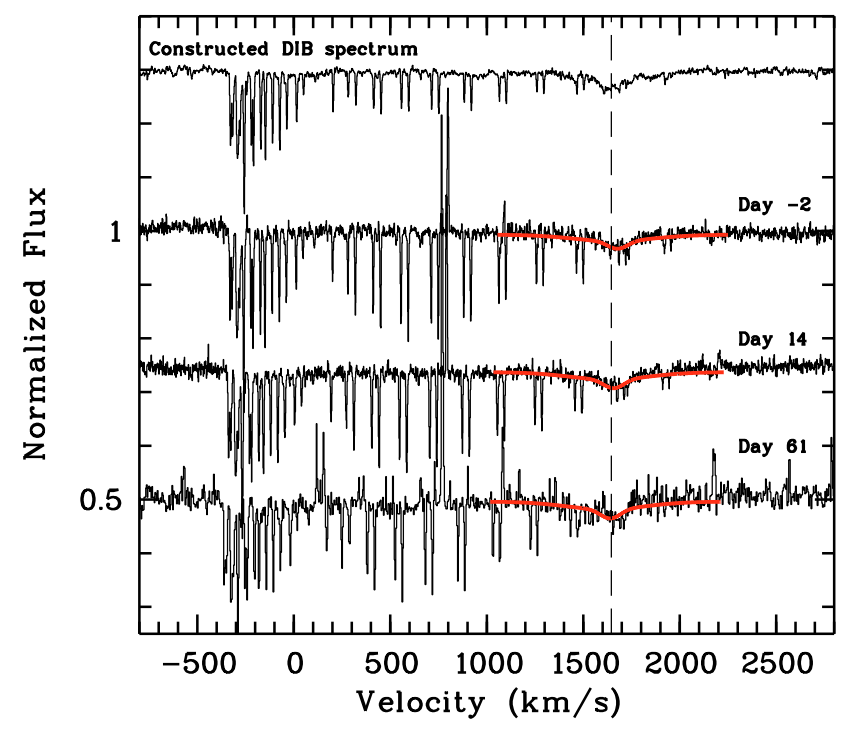

Fig. 5. The $6283.85 \AA$ DIB is detected toward SN2006X. The reference spectrum (top) has been constructed from the Galactic 6283 band profile of $\beta^{1}$ Sco and a Galactic telluric (unreddened) standard spectrum. The spectrum at phase -2 is rebinned to $2 \mathrm{~km} \mathrm{~s}^{-1}$ and to $4 \mathrm{~km} \mathrm{~s}^{-1}$ at +61 days. Vertical dashed line at $v_{\text {helio }}=1645 \mathrm{~km} \mathrm{~s}^{-1}$. 


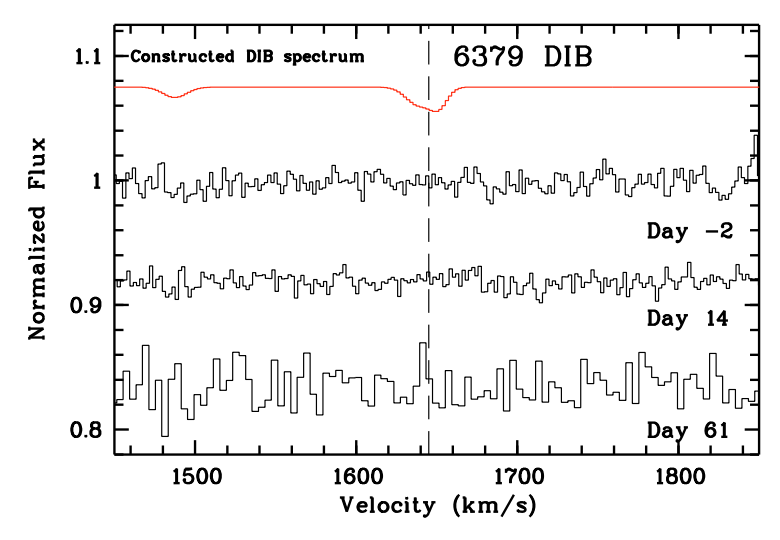

Fig. 6. The 6379.6 ̊ DIB is not detected toward SN2006X. The reference spectrum (top) has been constructed from the Galactic 6379 band profile toward $\sigma$ Sco shifted to the SN2006X velocity. Spectra at phase -2 and +14 are rebinned to $2 \mathrm{~km} \mathrm{~s}^{-1}$, and the spectrum at phase +61 to $4 \mathrm{~km} \mathrm{~s}^{-1}$. Vertical dashed line at $v_{\text {helio }}=1645 \mathrm{~km} \mathrm{~s}^{-1}$.

Table 4. Comparison of DIB strengths (in $\mathrm{m} \AA$ ) toward SN2006X in M 100, the Galaxy and LMC. Upper limits for $\lambda \lambda 6379$ and $6613 \mathrm{ob}-$ tained from weight averaged spectra at phase -2 and +14 . Equivalent widths for DIBs toward $\beta^{1}$ Sco and $\rho$ Oph from Sollerman et al. (2005) and for Sk-69 223 from Cox et al. (2006). Taurus dark cloud toward HD 283809 from Adamson et al. (1991).

\begin{tabular}{lllllll}
\hline \hline DIB & SN2006X & MW & $\beta^{1}$ Sco & $\rho$ Oph & Sk-69 223 & HD 283809 \\
$E_{B-V}$ & $\sim 1$ & 1.0 & 0.22 & 0.32 & 0.35 & 1.6 \\
\hline 6196 & $14 \pm 4$ & 53 & 20 & 10 & 10 & 46 \\
6283 & $177 \pm 25$ & 900 & 390 & 111 & 225 & 312 \\
6379 & $\lesssim 8$ & 88 & 14 & 24 & 55 & - \\
6613 & $\lesssim 18$ & 210 & 40 & 43 & 19 & - \\
\hline
\end{tabular}

subsequent determination of $E_{B-V}$ and $R_{V}$ (e.g. Wang 2005; Patat et al. 2006), provided that dust can survive the strong radiation field generated by the explosion. More and more supernova studies reveal dust with very low $R_{V} \sim 1-2$ (see for example Krisciunas et al. 2000, Elias-Rosa et al. 2006, 2008; Wang et al. 2006; Nobili \& Goobar 2007). It has not been established yet if the observed peculiar dust extinction is directly connected to the supernovae events or are representative of the extra-galactic ISM in the host galaxies.

Whether the remaining under-abundance of DIBs with respect to dust extinction is due to a lower gas-to-dust ratio (i.e. relatively less gas and more dust in the ISM), lower metallicity (i.e. not enough carbonaceous material to build DIBs) and/or a hard radiation field (i.e. a higher DIB destruction rate) is impossible to conclude without additional information on this line-ofsight (see for example Cox \& Spaans 2006).

One tantalizing possibility is that the line-of-sight toward SN2006X probes a dense interstellar cloud whose interior is shielded from the UV radiation and shows increased grain growth. Adamson et al. (1991) found a remarkable decline in DIB strength (per unit reddening) with increasing extinction (see Table 4 for values for the dark cloud toward HD 283809). It is unclear if the reduced strength of DIB features in dark clouds points to a grain or gas-phase related carrier.

Infrared/sub-mm data shows that M 100 is a typical spiral galaxy with a metallicity close to solar, a Galactic like PAH-todust mass fraction and a dust-to-gas mass ratio about twice that of the Galaxy (Draine et al. 2007). From this point of view, keeping in mind it is an overall average of the entire M 100 galaxy, there is twice the amount of dust compared to gas in M 100

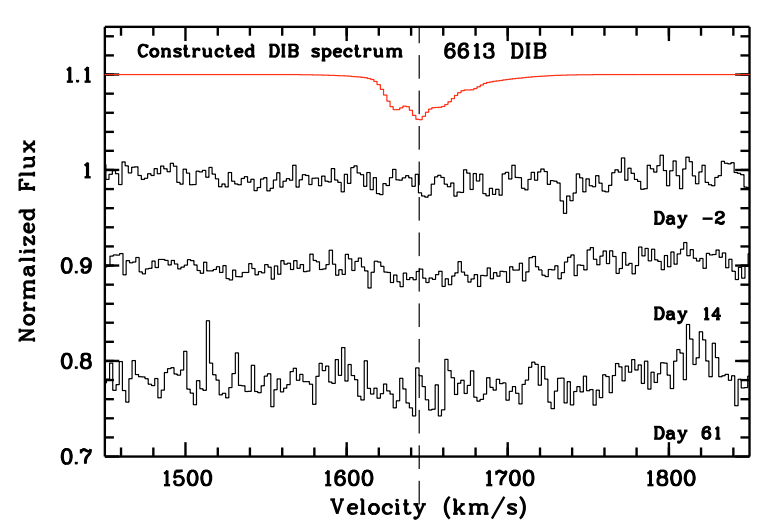

Fig. 7. The 6613.6 ̊ DIB is not detected toward SN2006X. The reference spectrum (top) has been constructed from the Galactic 6613 band profile toward $\sigma$ Sco shifted to the SN2006X velocity. Spectra are rebinned to $2 \mathrm{~km} \mathrm{~s}^{-1}$. Vertical dashed line at $v_{\text {helio }}=1645 \mathrm{~km} \mathrm{~s}^{-1}$.

than in the Milky Way (thus also the lower reddening inferred from $\mathrm{CH}$ and $\mathrm{CN}$ ). This could have a further effect, such as a decrease, on the DIB strength versus reddening relation.

Optically bright supernovae events offer not only a unique opportunity to study supernovae themselves and their immediate surroundings but also provide powerful background sources for the study of the diffuse to dense interstellar medium of galaxies beyond the Local Group that is otherwise inaccessible.

Acknowledgements. This research has made use of NASA's Astrophysics Data System and the SIMBAD database, operated at CDS, Strasbourg, France.

\section{References}

Adamson, A. J., Whittet, D. C. B., \& Duley, W. W. 1991, MNRAS, 252, 234 Ballester, P., Modigliani, A., Boitquin, O., et al. 2000, The Messenger, 101, 31 Benetti, S., Patat, F., Turatto, M., et al. 1994, A\&A, 285, L13 Cordiner, M. A., Cox, N. L. J., Trundle, C., et al. 2008, A\&A, 480, L13 Cox, N. L. J., \& Spaans, M. 2006, A\&A, 451, 973

Cox, N. L. J., Cordiner, M. A., Cami, J., et al. 2006, A\&A, 447, 991 Crawford, I. A. 1989, MNRAS, 241, 575

Dekker, H., D’Odorico, S., Kaufer, A., Delabre, B., \& Kotzlowski, H. 2000, in ed. M. Iye, \& A. F. Moorwood, Proc. SPIE, 4008, 534

D’Odorico, S., di Serego Alighieri, S., Pettini, M., et al. 1989, A\&A, 215, 21

Draine, B. T., Dale, D. A., Bendo, G., et al. 2007, ApJ, 663, 866

Elias-Rosa, N., Benetti, S., Cappellaro, E., et al. 2006, MNRAS, 369, 1880

Elias-Rosa, N., Benetti, S., Turatto, M., et al. 2008, MNRAS, 384, 107

Ellison, S. L., York, B. A., Murphy, M. T., et al. 2007, MNRAS, L124

Falgarone, E., Pineau des Forets, G., \& Roueff, E. 1995, A\&A, 300, 870

Fitzpatrick, E. L., \& Massa, D. 2007, ApJ, 663, 320

Krisciunas, K., Hastings, N. C., Loomis, K., et al. 2000, ApJ, 539, 658

Kuno, N., Sato, N., Nakanishi, H., et al. 2007, PASJ, 59, 117

Lauroesch, J. T., Crotts, A. P. S., Meiring, J., et al. 2006, CBET, 421, 1

Lawton, B., Churchill, C. W., York, B. A., et al. 2007, ArXiv e-prints, 711

Nobili, S., \& Goobar, A. 2007, ArXiv e-prints, 712

Patat, F., Benetti, S., Cappellaro, E., \& Turatto, M. 2006, MNRAS, 369, 1949

Patat, F., Chandra, P., Chevalier, R., et al. 2007, Science, 317, 924

Rand, R. J. 1995, AJ, 109, 2444

Rich, R. M. 1987, AJ, 94, 651

Sollerman, J., Cox, N., Mattila, S., et al. 2005, A\&A, 429, 559

Steidel, C. C., Rich, R. M., \& McCarthy, J. K. 1990, AJ, 99, 1476

Vidal-Madjar, A., Andreani, P., Cristiani, S., et al. 1987, A\&A, 177, L17

Vladilo, G., Crivellari, L., Molaro, P., \& Beckman, J. E. 1987, A\&A, 182, L59

Vladilo, G., Centurion, M., de Boer, K. S., et al. 1994, A\&A, 291, 425

Wang, L. 2005, ApJ, 635, L33

Wang, L., Baade, D., Höflich, P., et al. 2006, ApJ, 653, 490

Wang, X., Li, W., Filippenko, A. V., et al. 2008, ApJ, 675, 626

Welty, D. E., Frisch, P. C., Sonneborn, G., \& York, D. G. 1999, ApJ, 512, 636

Weselak, T., Galazutdinov, G. A., Musaev, F. A., \& Krełowski, J. 2004, A\&A, 414, 949 